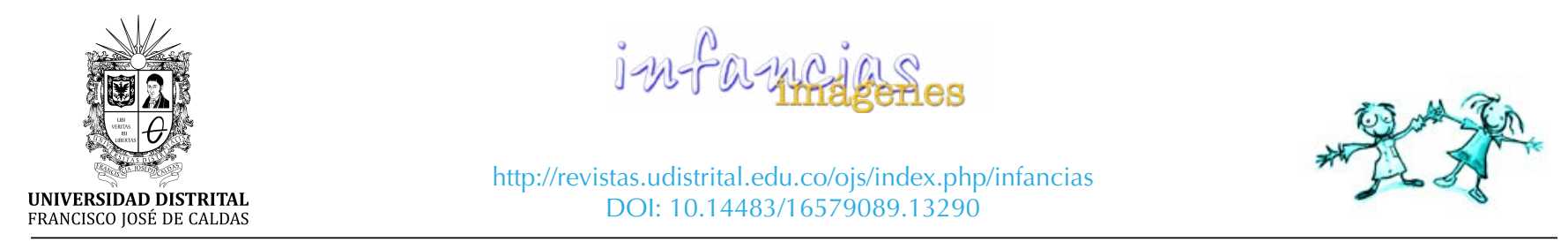

TEXTOS Y CONTEXTOS

\title{
La lectura en voz alta como estrategia para la comunicación adulto-niño en la escuela*
}

\section{Reading alloud as a strategy for adullt-child communication in school}

\author{
Elsa Ivonne Valencia Chaves', Alexandra Martínez Alzate²
}

Para citar este artículo: Valencia, E. I.; Martínez, A. (2018). La lectura en voz alta como estrategia para la comunicación adulto-niño en la escuela. Infancias Imágenes, 18(1), 142-149

\section{Resumen}

La materia Vivencia Escolar, dictada en segundo semestre de la licenciatura en Pedagogía Infantil de la Universidad Distrital, gira en torno al campo problé-
Recibido: 30-abril-2018 / Aprobado: 31-julio-2018

\begin{abstract}
The subject "School Experience", issued in the second semester of the degree in Child Pedagogy of the Universidad Distrital, revolves around the problematic area called adult-child communication, in which practice and other subjects established for the semester come together. That's why students must develop a field work with children of district school institutions that allows the exploration of those existing forms of communication between children with their peers, their teachers and themselves. This pedagogical exercise is developed through reading aloud as a transversal axis, based on interests and training needs of children; as well as the knowledge, pedagogical interests and learning development of our students. Reading aloud is thus a strategy that mediates other processes, since it's a real communicative space where they can relate to their peers and adults.
\end{abstract}

Keywords: oral reading, communication, educational sciences, training, teaching strategies.

Palabras clave: lectura oral, comunicación, pedagogía, formación, estrategia de enseñanza.

* Artículo de reflexión sobre los campos de la infancia, el lenguaje y la educación que no proviene de investigación. Ejercicio realizado durante 2014-II a 2016-II.

1 Licenciada en Lingüística y Literatura. Magíster en Estudios semiológicos. Magíster en Enseñanza del Español como Lengua Extranjera. DEA en América Latina Contemporánea, Instituto Universitario Ortega y Gasset. Profesora de vinculación especial en el área de lenguaje de la Licenciatura en Pedagogía Infantil, Universidad Distrital Francisco José de Caldas. Correo electrónico: mastereleivonne@gmail.com

2 Profesora de vinculación especial en el área de lenguaje de la licenciatura en Pedagogía Infantil, Universidad Distrital Francisco José de Caldas. Correo electrónico: alexafoca@yahoo.es 
"Al leer, lo importante no es lo que el texto dice, aquello a lo que el texto se refiere, sino lo que el texto nos dice, aquello hacia donde el texto se dirige. No se trata de desplegar un saber sobre un texto, sino de hacer la experiencia del texto"

Jorge Larrosa (s.f., p. 11)

Fin de partida. Leer, escribir, conversar ( $y$ tal vez pensar) en la universidad que viene Universidad de Barcelona, España.

\section{Introducción}

Estamos convencidas que todos recordamos a muchos de nuestros profesores quienes nos hechizaron en diversos instantes, como una especie de magnetismo sin explicación, que nos permitió sobrevivir a la escuela. Somos parte de ese sistema educativo que, en muchos casos, por no decir que en la mayoría, lograron el objetivo de la homogenización negándonos por completo a la alegría de disfrutar el aprendizaje de una manera distinta.

Para quienes aún transitamos por la academia, con múltiples labores de escritura, lecturas, discusiones y reuniones, aquellos profesores que nos hechizaron hicieron crecer en nosotros el gusto por la lectura y la escritura como forma de liberación de un mundo que nos lleva a ser todos iguales y a creer en la posibilidad de las trasformaciones sociales.

Por ello, la tarea de lectura y la exigencia que hacemos a nuestros estudiantes sobre la importancia de leer se trasforma, desde nuestra mirada, en el cambio de sentido hacia este proceso. Es decir, ¿por qué no comenzar utilizando como estrategia la lectura en voz alta para permitir a nuestros estudiantes soñar e imaginar? Esta estrategia la podemos usar los maestros para que nuestros niños y niñas encuentren otro camino hacia el aprendizaje y la autonomía, lo que les permitirá encontrarse con los otros a través de la lectura, la cual reivindica Pennac cuando afirma que:

El hombre que lee en viva voz se expone absolutamente a los ojos que lo escuchan... Si lee realmente, si pone en ello su saber controlando su placer, si su lectura es un acto de simpatía tanto para el auditorio como para el texto y su autor, si consigue hacer entender la necesidad de escribir despertando nuestras más oscuras necesidades de comprender, entonces los libros se abren de par en par, y la multitud de los que se creían excluidos de la lectura se precipita detrás de él. (2001, p. 155)

De esta manera, tiene sentido que para abordar el trabajo con las estudiantes de segundo semestre, se asuma al ser humano como un sujeto social y cultural que se construye y reconstruye a través del lenguaje, del pensamiento y de diversas formas de simbolizar. Los anteriores, le permiten representar y ver el mundo de modos particulares y acudir a esas significaciones para llevar a cabo sus interacciones con otros, dentro de esos diversos espacios sociales y culturales que habitan el mundo físico.

Por consiguiente, es importante que como profesores y formadores de profesores hablemos de un tema central que está presente día a día en nuestra existencia: la lectura en voz alta. Estrategia que constituye y da forma a la materia de Vivencia Escolar I en la que los estudiantes de II semestre se sumergen en ese otro espacio sociocultural que es la escuela, para establecer unas relaciones pedagógicas con los niños y poder, desde allí, vivir la experiencia de lo que acontece en la comunicación adulto-niño que es el eje articulador de este trabajo pedagógico.

Esta vivencia parte de un ejercicio propio con la lectura en voz alta realizado por las estudiantes, en el cual se buscaba que lograsen acercarse de manera directa a lo que significa vivir la lectura como un proceso que más allá de la decodificación de las palabras. Este ejercicio posibilita que ellas se conecten con el mundo cultural compartido con otros y con el mundo individual que construyen a través de las relaciones tejidas con los universos que emergen del encuentro. Así, pasamos a generar acercamientos con los espacios escolares donde los universos son los niños, con quienes se llevan a cabo acciones pedagógicas que los inviten a sumergirse en lo que significa ser cultura y significar el mundo gracias a los otros y la lectura compartida.

\section{Discusión}

La primera discusión que surgió fue la de qué comprendemos por lectura en la infancia y el papel que juega la lectura en voz alta como estrategia para comprender la comunicación adulto-niño. 
Categorías complejas que pueden verse desde diferentes perspectivas.

La idea inicial que se discutió en torno a lo que comprendemos por lectura, fue la de concebirla como un proceso. Si esto es así, implica que se realiza a lo largo de la vida de los seres humanos, y, por tanto, debe fomentarse y acompañarse en la escuela. La lectura es un proceso social y cultural de comprensión que permite relaciones e interacciones y que determina de algún modo a los sujetos en el mundo. Pero pensar la lectura en la infancia requiere observar, analizar y tener en cuenta varios factores por cuanto no se queda en el ideal de lo que significa leer, sino que hace énfasis en un grupo social particular que tiene unas características, necesidades e intereses distintos al resto de la sociedad.

Por lo anterior, si comprendemos la lectura en general como proceso de comprensión social, cultural, socializadora y que permite la construcción del sujeto, entonces la lectura en la infancia debería, en principio, poseer las características mencionadas y otras particularidades que hacen que hablemos de lectura en la infancia. Varios autores han mostrado la importancia de la lectura en la infancia desde el desarrollo de los procesos cognitivo en los niños y las niñas, así como el fortalecimiento de lo socioemocional y el papel que juega en el proceso de aprendizaje en la familia y la escuela.

Desde esta perspectiva, nuestra discusión se dio, al inicio de la propuesta, sobre lo que comprendemos por ella. Consideramos en nuestra reflexión que la lectura en la infancia representa un campo en el que se ha trabajado desde lo teórico, pero, que ha sido dejado de lado en la escuela y en las aulas donde habitan los niños y niñas.

La lectura en la infancia representa una dimensión que creemos debe ser incluida de manera natural en la escuela, como forma de contribuir a la construcción de los sujetos. De esta manera, el papel que juega en el desarrollo de los niños y niñas deberá ser parte fundamental de los planes de aula que realizan los maestros de los diferentes grados escolares como parte del proceso de enseñanza y aprendizaje infantil.

Es así como la lectura en la infancia, al insertarse en los programas escolares, debe ser pensada en término de tipologías de lecturas, formas de leer en el aula, disfrute por el proceso de leer y otros aspectos que deben ser reflexionados por los maestros como lo son la importancia y el impacto de este proceso del lenguaje en la escuela y de las estrategias a utilizar para que el proceso de comprensión y disfrute de la lectura en la infancia sea posible.

Por tanto, en el espacio de Vivencia Escolar con las estudiantes de segundo semestre de la licenciatura en Pedagogía Infantil de la UDFJC, cuyo eje de reflexión es la comunicación adulto-niño, consideramos que utilizar la estrategia de lectura en voz alta (LVA) como mediación para el desarrollo y fortalecimiento de procesos de comprensión, así como la disculpa para acercarnos a las formas que utilizan estos sujetos para comunicarse, representa una posibilidad que puede contribuir a que nuestros niños y niñas no solamente realicen procesos cognitivos exigidos en el espacio escolar, sino que, además, aporte en el fortalecimiento de relaciones más cercanas y horizontales con el adulto a partir de las formas de comunicar.

Reflexionar en torno a estas categorías nos Ilevó a considerar que desde los diferentes espacios académicos se involucren las realidades que viven los maestros en formación, lo cual permite la reflexión, análisis y configuración de propuestas sustentadas en el reconocimiento de los sujetos escolares como participantes activos de un proceso de cambio y trasformación hacia el reconocimiento del otro en su diferencia y diversidad para comunicarse.

Por consiguiente, abordar la lectura en voz alta dentro de las aulas de clase como la estrategia central de las planeaciones reconfigura la esencia del espacio escolar, ya que lo asume como parte de la cultura y a los niños, maestros y vivencialistas como sujetos sociales que se construyen y reconstruyen dentro de esa esfera comunicativa; que otorga oportunidades de ser escuchados, leídos y acogidos.

\section{Propuesta \\ Inicio}

Dentro del espacio académico de Vivencia Escolar, hemos considerado necesario dialogar con los estudiantes respecto a su papel como formadores dentro de una educación pública; así como su responsabilidad y compromiso al pertenecer a un espacio en el que se debe fortalecer la concepción de 
la educación como un derecho de los sujetos y una obligación del Estado.

Por tanto, se reflexiona en un primer momento en torno a la necesidad de mejorar los niveles de pensamiento crítico, el trabajo autónomo real y la competencia comunicativa de los estudiantes, a partir de la estrategia de la lectura en voz alta como eje trasversal, además del papel que se tiene como maestro en la construcción de una sociedad más igualitaria y equitativa.

Así mismo, el conocimiento de saberes disciplinares, el manejo de estrategias pedagógicas, didácticas y metodológicas junto a la reflexión práctica de quiénes debemos ser como formadores de la infancia en Colombia, están presentes en los diferentes discursos que circulan en el aula. Es decir, este espacio se convierte en observatorio de las diferentes prácticas comunicativas que realizan los sujetos adultos-niños, teniendo como hilo de tejido el lenguaje y su uso en el entorno escolar.

Con lo anterior, dentro de la situación formal de desarrollo de Vivencia Escolar II, las profesoras formadoras organizamos este espacio académico con dos objetivos: el primero pretende dar recursos interdisciplinares para que las estudiantes establezcan coordenadas cognitivas respecto a lo que implica el lenguaje y la comunicación dentro del desarrollo de la infancia en distintos ámbitos. Por tal motivo, se ejecutan una serie de actividades posibilitadoras que Ileven a las vivencialistas a proponer su plan de aula siguiendo el enfoque por tareas. El segundo objetivo busca que puedan de manera autónoma explorar el entorno escolar e insertar ideas a la propuesta de aula, comprendiendo que a través de la lectura en voz alta pueden tener el pretexto para proponer un programa de aprendizaje enfocado en el uso real y auténtico de la lengua en el aula por parte de los niños, niñas y las vivencialistas mismas.

Se parte del principio del reconocimiento del sujeto-niño en su realidad individual, social y cultural; medio eficaz que permite dos procesos fundamentales en el desarrollo de la competencia comunicativa y en el establecimiento de relaciones más saludables y cercanas entre los actores que intervienen en el proceso de comprensión auditiva y el proceso de producción oral.
Las acciones que permiten el logro de los objetivos trazados se inician un mes antes de ir a la institución escolar durante el espacio de Seminario. Por una parte, se realiza la lectura de autores que trabajan sobre el ejercicio de observación en el aula, para ello, realizamos una primera visita de observación holística a la institución para conocer el contexto institucional, hablar con las maestras y comenzar a pensar lo que significan las dinámicas escolares; centrando la atención en el uso del diario de campo como herramienta metodológica que permite no solo comprender el contexto escolar, sino, sobre todo, evidenciar que la práctica pedagógica debe ser una permanente reflexión acerca de nuestra práctica de enseñanza.

Por otra parte, se realiza un ejercicio en el que cada equipo de vivencialistas debe proponer la lectura de un texto de la literatura infantil de acuerdo con el grado escolar que se le asigne para su implementación. Antes de realizar el ejercicio, deben entregar un escrito en el que justifican las razones por las que escogieron el texto para la LVA y disponerse para escenificar el ejercicio planteado ante sus compañeros de seminario y las docentes quienes observan y toman notas sobre la práctica realizada. Al finalizar, se realiza una retroalimentación teniendo en cuenta una serie de criterios asociados a las formas de leer, la pertinencia del texto para un determinado público, la organización de materiales, espacios, escenografías y el dominio sobre el texto seleccionado, entre otros.

El ejercicio práctico y la retroalimentación posterior tienen como propósito pedagógico que las estudiantes de Vivencia Escolar reflexionen acerca de sus creencias y acciones sobre lo que significa leer en voz alta a los niños y niñas, selección del texto, maneras de comprender la práctica pedagógica y lo que asumen como didáctica. Esta reflexión se sustenta en diferentes autores que han trabajado sobre el uso de la estrategia de la LVA en el aula teniendo en cuenta diferentes aspectos, que siguiendo a Rodríguez, Gaviria y Garrido (2016) nos permite desarrollar otros procesos cognitivos y socioemocionales tales como:

- La mediación fónica del texto escrito, como artefacto auditivo. 
- La entonación del discurso como paso previo a la comprensión. El texto escrito es un artefacto incompleto que necesita de la mediación del lector para completar el sentido gracias a la identificación de sus unidades nocionales.

- La LVA es una estrategia que permite entrenarnos para comprender al otro, a los adultos y a sus pares. Es la disculpa que utilizamos para acercarnos a los niños y niñas y comprender esas otras formas que utilizan para comunicarse y expresar sentidos.

- La dimensión emocional de quienes participan a través de la escucha activa y el fortalecimiento de la tolerancia, el respeto y el silencio como forma de compartir.

Lo anterior se articula al resto del trabajo que se realiza en el seminario alrededor de lo que implica una planeación de aula, la construcción de un objetivo general que se ve trasformado en la medida en que se encuentran con los niños y niñas en el aula, los objetivos específicos, los tipos de lecturas a utilizar, las diferentes tareas posibilitadoras que apoyan una práctica comunicativa, con la intención de que las estudiantes comprendan que el papel del maestro va más allá de los contenidos (al asumir las necesidades e intereses de los niños y niñas como ingredientes fundamentales en el proceso de desarrollo comunicativo de ellos).

Por consiguiente, se tiene en cuenta antes de ir a la práctica real la selección de los textos, los materiales que se emplean, formatos a trabajar, contenidos temáticos, nivel lingüístico, sociolingüístico, sociocultural, pragmático de los niños y las tareas que los niños van a desarrollar respecto a la lengua utilizada en LVA. Es decir, se enfatiza en características como: pronunciación, vocalización, entonación con oscilaciones, vocabulario adecuado para la edad de los niños, frases completas y acordes al nivel gramatical de los niños, tipo de imágenes, entre otros.

De esta manera, la LVA no se comprende como leer para ser escuchados, sino que, por el contrario, se plantea como una estrategia a utilizar teniendo en cuenta componentes pedagógicos y didácticos que posibiliten la comunicación entre las vivencialistas, los niños y niñas. Lograr esto nos permitirá, en la práctica en el aula, comprender que la LVA es una estrategia que posibilita en todos nosotros (niños, niñas y adultos) ir más allá de la simple decodificación del código, para avanzar en la construcción de otras formas de comprender el mundo. Bien lo dice Leopoldo Brizuela cuando nos invita a pensar que:

por alguna razón, pensamos la lectura como algo personal, íntimo, cuando no tiene por qué ser así; [...] la literatura podría ganar muchos lectores si la gente tuviera oportunidad de reunirse a leer. La lectura en voz alta es el modo de lograr que cada estudiante pueda leer y leerse, solo, después, en la intimidad: ese diálogo con uno mismo que, según Hanna Arendt, es la gran conquista del espíritu humano. (2009, p. 30)

La LVA no es asumida como una simple narración de cuentos. Bien lo explica Chambers (1999) cuando establece una clara diferenciación de esta con la narración de cuentos al plantear que la LVA permite objetivar la experiencia. Con esta afirmación, hemos considerado importante realizar una reflexión sobre aquellos temas de interés en los niños ya que, en últimas, lo que nos importa es quienes escuchan, aquellos que se incluyen para ser reconocidos y auto-reconocerse como sujetos de comunicación y lenguaje; descubriendo como resultado que su lenguaje se valida en los procesos de intercambio con el adulto.

Incluir la LVA en la escuela es una forma de fortalecer la dimensión socioemocional en los niños y niñas encuanto posibilita que las relaciones entre el adulto y el niño y sus formas de comunicación nos lleven a nuevas formas de configurar la realidad. Bien lo dice Yolanda Reyes cuando afirma que:

la inclusión de la lectura en la primera infancia, a través de la tradición oral y de los textos de la cultura (literatura, música, juego), fomenta la comunicación, imprimiéndole una carga afectiva que fortalece los vínculos, enriquece y resignifica los patrones de crianza y se constituye en poderosa herramienta de prevención emocional. (2005, p. 10) 
Por tanto, la LVA se organiza de acuerdo con las características, necesidades e intereses de los grupos. Esto da la posibilidad, por un lado, de que lean los textos desde la diversidad de lenguajes que los niños manejan: imágenes visuales, evocaciones que ellas les generan, las asociaciones que establecen ya sean por el color, el tamaño, la forma del texto y los que surgen dentro del grupo; y, por otro lado, de realizar ejercicios de interacción y comunicación más complejos entre los niños y las vivencialistas.

De este modo, se reconfiguran los espacios, las relaciones entre adulto-niños y se valida al lenguaje y en especial a la lectura en voz alta como una posibilidad de ser sujetos críticos, creadores, comunicantes $y$, por ende, culturales que se asumen como textos al significar y resignificar su sentido del mundo a través del lenguaje y sus procesos.

\section{Durante}

Una vez finalizada esta primera etapa que se gestiona en el primer mes del semestre, las vivencialistas acompañan durante una jornada semanal al curso correspondiente, Ilevan a cabo su planeación con los niños y niñas acompañadas siempre por la docente encargada del curso, hacen parte activa de las diferentes dinámicas institucionales y entregan su planeación reestructurada de acuerdo con lo que vaya sucediendo cada semana. Así mismo, se desarrolla el seminario semanalmente en el que se reflexiona, discute y analizan los diferentes autores propuestos en el syllabus, el diario de campo y las planificaciones de aula diseñadas para el trabajo.

La planeación, que en la etapa de inicio se entrega y se ha discutido durante el primer mes, se rediseña y orienta de acuerdo con cada grupo de niñas y niñas. Es así que la propuesta inicial se ve trasformada y nutrida por las estudiantes a lo largo de la Vivencia Escolar, quienes en principio deben tener en cuenta no solo las observaciones realizadas durante el proceso por las directoras de grupo, las docentes de la universidad que las acompañamos, sino, además, sus reflexiones y apuestas que realizan en la experiencia con los niños y niñas en la institución.

Las diferentes lecturas que se realizan dentro del seminario, permiten que las estudiantes no solo den cuenta de la propuesta de los diversos autores que se abordan, sino que sirven para que se reflexione en torno a lo que es trabajar con y para la infancia de Bogotá. Los autores seleccionados tienen que ver con el papel del maestro, la lectura como habilidad a fortalecer, el desarrollo cognitivo y socioafectivo en los niños, la planeación pedagógica, el diario de campo y la LVA como estrategia de enseñanza.

\section{Final}

La planeación que realizan las estudiantes se ve trasformada por las relaciones que se dan al interior de la institución. De acuerdo con la experiencia, las planeaciones que se llevan a cabo se cruzan con las actividades o sucesos que surgen en el hacer diario del colegio. Lo anterior significa que las estudiantes se ven expuestas no solo a comprender a los niños como universos que se encuentran en continuo cambio, sino a la misma institución y a todo lo que acontece dentro de ella como razones para pensar en la educación como un ir y venir con sentido y conexión.

Por tanto, una vez finalizada la vivencia escolar en el colegio, las estudiantes deben realizar el ejercicio de reflexión escrita. Esto, en una estructura de informe en el que deben hablar sobre la experiencia personal, la relevancia de los autores trabajados respecto a lo que significa la comunicación adulto-niño y el papel del maestro que trabaja con la infancia, así como el papel de las instituciones y las comprensiones, tensiones y preguntas respecto a los procesos educativos, sociales, culturales, políticos y comunicativos que se tejen dentro de los espacios escolares.

\section{Conclusiones}

Lograr que las estudiantes al finalizar el semestre académico se encuentren en un proceso de reflexión sobre su propio proceso pedagógico y sus formas de enseñanza como maestros, es un propósito que las docentes que lideramos este espacio académico perseguimos sembrar en ellas para la vida. Propósito que implica llevar a cabo en comunidad discusiones que tienen que ver con la decisión voluntaria de dedicar la vida a la enseñanza y acompañamiento escolar a nuestra infancia; y no 
con el único argumento que esgrimen al inicio del semestre cuando enuncian las razones, en la mayoría de los casos, que tuvieron para iniciar la licenciatura en Pedagogía Infantil: "es que me gustan los niños".

Intentar mostrar esta mirada a nuestras estudiantes sobre las responsabilidades y compromisos que se adquieren cuando se toma este camino forma parte del eje que atraviesa la Vivencia Escolar I. Lo anterior, en cuanto a que ellas se dan cuenta en la experiencia compartida cada semana con sus cursos a cargo, que la tarea de ser maestro de niñas y niños requiere mucho más que el "gusto", pues es una labor que exige la formación disciplinar, pedagógica y didáctica.

Abordar el eje de la Vivencia Escolar I desde la perspectiva de comprender a los otros como sujetos de comunicación ha posibilitado experiencias en el aula que nos muestran que vamos por buen camino, hacia la comprensión de lo que implica los diferentes procesos involucrados en el ejercicio docente.

El uso de la estrategia de LVA, como excusa para comprender las diversas maneras de comunicación que se pueden dar en la escuela entre adultos y niños, muestra la importancia de comprender el ejercicio de planeación como un proceso de largo aliento ya que es una parte del engranaje de la configuración como maestro.

Durante el camino que recorren nuestras estudiantes en el proceso de planeación de aula, lectura de textos que fundamentan la experiencia, estar de nuevo en la escuela desde la otra orilla, ser responsable de aproximadamente 30 niños, participar en los eventos institucionales y ser responsables de entregar lo que se solicita desde lo académico, ha sido una experiencia para ellas que les permite, al finalizar el semestre (lo cual se evidencia en el informe escrito y la sustentación oral), una reflexión consciente de los compromisos y responsabilidades que adquieren si deciden continuar la licenciatura.

Las estudiantes, cuando inician el ejercicio de LVA, consideran que es una tarea fácil por cuanto ellas saben leer. Sin embargo, cuando realizan el proceso de comprender lo que implica y la experiencia de su realización en el aula advierten que la LVA es mucho más que la decodificación del código para pasar a ser un proceso de construcción y configuración de sentido entre todos los que participan. Por consiguiente, la lectura en voz alta resignifica el trabajo en las aulas de clase al reconocer a los niños y niñas como sujetos culturales que se construyen a través del lenguaje y la comunicación.

Así mismo, la lectura en voz alta posibilita la reflexión situada de las estudiantes en formación respecto a la infancia, la comunicación y los entornos escolares. A través de la lectura en voz alta se abordan y fortalecen una serie de componentes de la competencia comunicativa, fundamentales para la interacción social y para el desarrollo de procesos cognitivos y emocionales.

Así es como la Vivencia Escolar I contribuye a generar en las estudiantes reflexiones argumentadas respecto a los procesos de comunicación dados dentro de los espacios escolares, gracias a que a través de la LVA se resignifican las prácticas pedagógicas al conceptualizar a la educación como un proceso de construcción y reconstrucción del mundo social, cultural y político. Planeación pedagógica en la que se asume a los niños como sujetos socioculturales que tienen un papel y un valor como sujetos comunicativos.

La selección de autores a trabajar dentro de la Vivencia Escolar I ha sido establecida por las necesidades de comprender la escuela, la educación, la pedagogía y el lenguaje como mecanismos, por así decirlo, que forman parte de una construcción colectiva que permite llevar a cabo una labor mucho más directa en las propuestas que se llevan al interior del aula.

Del mismo modo, estas selecciones están relacionadas con el campo problémico denominado comunicación adulto-niño, con el cual se pretende cohesionar la formación del segundo semestre y dirigir estas propuestas pedagógicas que las estudiantes desde su primera mirada a la escuela y las infancias que se mueven dentro de ella.

Por otra parte, se busca contribuir desde el espacio de seminario en el proceso de reflexión y contraste entre las formulaciones teóricas de los autores abordados y las realidades encontradas en los entornos escolares donde se lleva a cabo la vivencia. De esta manera, las estudiantes no solo vivirán la 
experiencia directa en las aulas sino que buscarán comprender a la luz de la teoría el porqué de muchas o algunas de las situaciones que se presentan en este espacio, entre los actores que lo habitan y, desde allí, se puedan plantear las múltiples formas en que ellas participarían de una trasformación de la escuela, de las relaciones entre el adulto y el niño, de los usos del lenguaje y posicionarse así como mediadoras y posibilitadoras de estos cambios desde su práctica.

El espacio académico de Vivencia Escolar I se ha preocupado por acercar a las estudiantes a la realidad escolar existente y a las infancias que viven en ella. Por lo tanto, bosqueja un plan de trabajo que les permite visibilizar la importancia y necesidad de reconocer a los sujetos y actores fundamentales de todo proceso educativo como participantes activos haciendo uso del diario de campo como medio para evidenciar las observaciones que realizan las estudiantes.

Finalmente, la Vivencia Escolar I no es solo un espacio académico, también es de formación personal pues les da la posibilidad a las estudiantes de autorreconocerse frente a la realidad de la educación colombiana y del distrito, a su vez que les provee de bases significativas alrededor del lenguaje y la comunicación. Esto no solo desde la teoría, sino desde la práctica, en la interacción con los niños y en los procesos de enseñanza y aprendizaje, como primer referente de lo que se van a encontrar en las demás vivencias.

\section{Referencias}

Brizuela, L. (2009). Leer en voz alta. Actas del $14^{\circ}$ Foro Internacional por el Fomento del Libro y la Lectura. Resistencia, Argentina: Fundación Mempo Giardinelli.

Chambers, A. (1999). Narración de cuentos y lectura en voz alta. Caracas: Banco del Libro.

Pennac, D. (2001). Como una novela. Barcelona: Anagrama.

Reyes, Y. (2005). La lectura en la primera infancia. Bogotá: Centro Regional para el Fomento del Libro en América Latina y el Caribe (Cerlalc).

Rodríguez, T, Gaviria, C. y Garrido, E. (2016). La lectura en voz alta. Programas clásicos escolares 15/16. Andalucía, España: Junta de Andalucía, Consejería de Educación. 